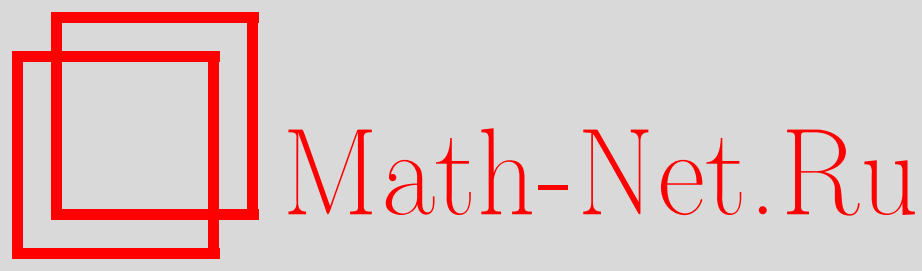

М. Эйлер, Н. Эйлер, С. Лундберг, Об обратимых преобразованиях Беклунда для автономных эволюционных уравнений, ТМФ, 2009, том 159, номер 3, 418-427

DOI: https://doi.org/10.4213/tmf6361

Использование Общероссийского математического портала Math-Net.Ru подразумевает, что вы прочитали и согласны с пользовательским соглашением http: //www . mathnet.ru/rus/agreement

Параметры загрузки:

IP: 35.173 .219 .149

26 апреля 2023 г., 10:58:07

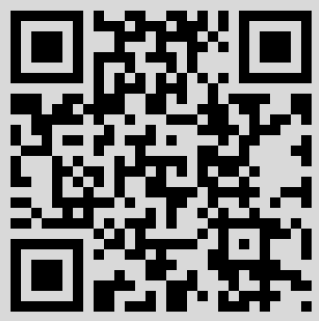




\section{ОБ ОБРАТИМЫХ ПРЕОБРАЗОВАНИЯХ БЕКЛУНДА ДЛЯ АВТОНОМНЫХ ЭВОЛЮЦИОННЫХ УРАВНЕНИЙ}

Обсуждается построение обратимых преобразований Беклунда для эволюционных уравнений с применением интегрирующих множителей нулевого и старших порядков и соответствующих им законов сохранения. В качестве примера рассматриваются уравнение Гарри Дима и уравнение Шварциана-КдФ.

Ключевые слова: нелинейное эволюционное уравнение, преобразование Беклунда, закон сохранения, интегрируемое эволюционное уравнение.

\section{1. ВВЕДЕНИЕ}

Как указано в недавней работе Роджерса и Шифа [1], обратимое преобразование Беклунда было предложено еще в статье Хаара [2]. Современный подход к обратимому преобразованию Беклунда и некоторым его приложениям можно найти в работе [3]. В настоящей работе выводятся обратимые преобразования Беклунда для эволюционных уравнений с использованием интегрирующих множителей и соответствующие им законы сохранения. Интегрирующие множители старших порядков приводят к законам сохранения старших порядков для уравнений. Показано, что эти законы сохранения можно использовать для задания обратимых преобразований Беклунда с интересными уравнениями, получающимися в качестве образа.

При построении интегрирующих множителей и законов сохранения мы следуем работам [4]-[8], применяя обозначения из работ [9], [10] (относительно симметрийных интегрируемых уравнений эволюции см. статьи [11], [12] и ссылки в этих работах). Показано, какое влияние оказывают различные интегрирующие множители старшего порядка при применении их к эволюционным уравнениям в рамках обратимого преобразования Беклунда. В деталях обсуждаются два примера эволюционных уравнений третьего порядка: уравнение Гарри Дима и уравнение Шварциана-КдФ.

*Department of Mathematics, Luleå University of Technology, SE-971 87 Luleå, Sweden. E-mail: Norbert.Euler@sm.luth.se 


\section{2. ПРЕОБРАЗОВАНИЯ}

Рассмотрим автономное эволюционное уравнение общего вида

$$
E:=u_{t}-F\left(x, u, u_{x}, u_{x x}, u_{3 x}, \ldots\right)=0
$$

и предположим, что оно имеет закон сохранения

$$
D_{t} \Phi^{t}\left(x, u, u_{x}, u_{x x}, \ldots\right)+D_{x} \Phi^{x}\left(x, u, u_{x}, u_{x x}, \ldots\right)=0,
$$

где $\Phi^{t}$ - ток и $\Phi^{x}$ - поток. Следуя работе [10], имеем

ПРЕДЛОЖЕНИЕ 1. Пусть $\Lambda$ - присоединенная симметрия уравнения (2.1), которая пролвляет себя в виде интегрирующего множителя для (2.1), т.е. $\Lambda$ удовлетворяет условию

$$
\left.L_{E}^{*}[u] \Lambda\right|_{E=0}=0,
$$

max чmo

$$
\widehat{E}(\Lambda E)=0,
$$

где через $\widehat{E}$ обозначен оператор Эйлера,

$$
\widehat{E}[u]:=\frac{\partial}{\partial u}-D_{x} \circ \frac{\partial}{\partial u_{x}}-D_{t} \circ \frac{\partial}{\partial u_{t}}+D_{x}^{2} \circ \frac{\partial}{\partial u_{x x}}-D_{x}^{3} \circ \frac{\partial}{\partial u_{3 x}}+\cdots,
$$

u $L_{E}^{*}[u]$ - оператор, сопряженный $к$ линейному оператору $L_{E}[u]$. Другими словами,

$$
\begin{aligned}
& L_{E}[u]:=\frac{\partial E}{\partial u}+\frac{\partial E}{\partial u_{t}} D_{t}+\frac{\partial E}{\partial u_{x}} D_{x}+\frac{\partial E}{\partial u_{x x}} D_{x}^{2}+\cdots+\frac{\partial E}{\partial u_{n x}} D_{x}^{n}, \\
& L_{E}^{*}[u]:=\frac{\partial E}{\partial u}-D_{x} \circ \frac{\partial E}{\partial u_{x}}-D_{t} \circ \frac{\partial E}{\partial u_{t}}+D_{x}^{2} \circ \frac{\partial E}{\partial u_{x x}}+\cdots+(-1)^{n} D_{x}^{n} \circ \frac{\partial E}{\partial u_{n x}} .
\end{aligned}
$$

Тогда соответствующий закон сохранения имеет вид

$$
D_{t} \Phi^{t}+D_{x} \Phi^{x}=0,
$$

где

$$
\Lambda=\widehat{E}[u] \Phi^{t}\left(x, u, u_{x}, u_{x x}, \ldots\right) .
$$

Рассмотрим два автономных эволюционных уравнения порядка $n \geqslant 2$ относительно соответствующих функций $u(x, t)$ и $v(y, \tau)$ :

$$
\begin{aligned}
& u_{t}=F\left(x, u, u_{x}, u_{x x}, \ldots, u_{n x}\right), \\
& v_{\tau}=G\left(y, v, v_{y}, v_{y y}, \ldots, v_{n y}\right) .
\end{aligned}
$$

Следуя работе [13], определим обратимое преобразование Беклунда $R$ следующим образом. 
ОПРеДЕЛЕНиЕ 1. Рассмотрим замену переменных при преобразовании $R$ : $(u, x, t) \mapsto(v, y, \tau)$, заданную следующим образом:

$$
\begin{aligned}
d y(x, t) & =\Phi^{t}\left(x, u, u_{x}, u_{x x}, \ldots\right) d x-\Phi^{x}\left(x, u, u_{x}, u_{x x}, \ldots\right) d t, \\
R: \quad d \tau(x, t) & =d t \\
v(y, \tau) & =g\left(x, u, u_{x}, \ldots\right),
\end{aligned}
$$

с законом сохранения для (2.10а) вида (2.2). Преобразование $R$ называется обратимъмм преобразованием Беклунда, если найдется второе преобразование $S:(v, y, \tau) \mapsto$ $(\tilde{u}, \tilde{x}, \tilde{\tau})$ вида

$$
\begin{aligned}
d \tilde{x}(y, \tau) & =\Psi^{\tau}\left(y, v, v_{y}, v_{y y}, \ldots\right) d y-\Psi^{y}\left(y, v, v_{y}, v_{y y}, \ldots\right) d t \\
S: \quad d \tilde{\tau}(y, \tau) & =d \tau \\
\tilde{u}(\tilde{x}, \tilde{\tau}) & =h\left(y, v, v_{y}, \ldots\right)
\end{aligned}
$$

с законом сохранения для уравнения (2.10б)

$$
D_{\tau} \Psi^{\tau}\left(y, v, v_{y}, v_{y y}, \ldots\right)+D_{y} \Psi^{y}\left(y, v, v_{y}, v_{y y}, \ldots\right)=0
$$

такое, что

$$
S \circ R=R \circ S=I,
$$

где $I$ - тождественное преобразование.

Следующее предложение напрямую следует из определения 1 и может быть применено для задания $g$ из (2.11) таким образом, чтобы преобразование $R$ оказалось обратимым преобразованием Беклунда.

ПреДЛОЖЕНИЕ 2. Уравнение (2.10а) тогда и толъко тогда допускает обратимое преобразование Беклунда $R(2.11)$ с преобразованием $S$ (2.12), которое связывает уравнения (2.10а) и (2.10б), когда

$$
\begin{aligned}
\left.\Psi^{\tau} \Phi^{t}\right|_{u \rightarrow v} & =1, \\
\Psi^{y}+\left.\Psi^{\tau} \Phi^{x}\right|_{u \rightarrow v} & =0,
\end{aligned}
$$

где функиия $h$ в (2.12) определена так, чтобъ выполнялось соотношение (2.14).

Заметим, что так называемое $x$-обобщенное преобразование годографа, введенное в работах [14], [15], одновременно оказывается обратимым преобразованием Беклунда в соответствии с определением 1. Примеры приведены в следующем разделе.

\section{3. ПРИМЕРЫ}

В этом разделе описано в деталях, как определение 1 и предложения 1,2 применяются к двум явным эволюционным уравнениям третьего порядка, а именно к уравнению Гарри Дима и к уравнению Шварциана-КдФ. С помощью обратимого преобразования Беклунда эти уравнения могут быть приведены к не зависящим от $x$ 
автономным эволюционным уравнениям. Это означает, что необходимо определить функцию $g$ в обратимом преобразовании Беклунда $R(2.11)$ так, чтобы получившееся при этом уравнение не зависело явно от $x$. Это налагает серьезные ограничения на вид функции $g$. Заметим также, что различные способы выбора функции $g$ приводят к различным результирующим уравнениям для одних и тех же сохраняющихся величин $\Phi^{t}$ и $\Phi^{x}$ (см., например, ниже случаи 1.1 и 1.2 для уравнения Гарри Дима).

3.1. Уравнение Гарри Дима. Рассмотрим уравнение Гарри Дима в следующем виде:

$$
u_{t}=u^{3} u_{3 x} .
$$

В силу предложения 1 полный класс автономных (не зависящих от времени) присоединенных симметрий вплоть до второго порядка, которые также действуют как интегрирующие множители для уравнения (3.1), имеет следующий вид:

$$
\begin{array}{cc}
\Lambda_{1}=\alpha u^{-2}, & \Lambda_{2}=\alpha u^{-3}, \quad \Lambda_{3}=\alpha x u^{-3}, \\
\Lambda_{4}=\alpha x^{2} u^{-3}, & \Lambda_{5}=\alpha\left(u^{-1} u_{x x}-\frac{1}{2} u^{-2} u_{x}^{2}\right) .
\end{array}
$$

Здесь $\alpha$ - произвольная константа, которая выбирается ниже подходящим образом. Применим эти интегрирующие множители, чтобы показать, как с помощью предложений 1, 2 могут быть определены различные обратимые преобразования Беклунда, и свяжем таким образом класс эволюционных уравнений с уравнением Гарри Дима (3.1).

СлУчАЙ 1. Рассмотрим $\Lambda_{1}=-u^{-2}$. Соответствующий не зависящий от $x$ ток нулевого порядка $\Phi^{t}$ и его поток $\Phi^{x}$ для уравнения (3.1) имеют вид

$$
\Phi^{t}(u)=u^{-1}, \quad \Phi^{x}\left(u, u_{x}, u_{x x}\right)=u u_{x x}-\frac{1}{2} u_{x}^{2} .
$$

СлУчАЙ 1.1. Пусть $g=x$; это означает, что преобразование $R(2.11)$ представляет собой пример $x$-обобщенного преобразования годографа, а именно

$$
\begin{aligned}
d y(x, t) & =u^{-1} d x-\left(u u_{x x}-\frac{1}{2} u_{x}^{2}\right) d t, \\
R: \quad d \tau(x, t) & =d t \\
v(y, \tau) & =x .
\end{aligned}
$$

Применяя преобразование $R$ к (3.1), получим уравнение Шварциана-КдФ

$$
v_{\tau}=v_{3 y}-\frac{3}{2} v_{y}^{-1} v_{y y}^{2} .
$$

Из предложения 2 следует, что (3.3) представляет собой обратимое преобразование Беклунда с преобразованием $S$ вида

$$
\begin{aligned}
d \tilde{x}(y, \tau) & =v_{y} d y-\left(-v_{3 y}+\frac{3}{2} v_{y}^{-1} v_{y y}^{2}\right) d t, \\
S: \quad d \tilde{\tau}(y, \tau) & =d \tau, \\
\tilde{u}(\tilde{x}, \tilde{\tau}) & =v_{y},
\end{aligned}
$$


таким образом, соответствующие сохраняющиеся ток и поток для (3.4) в данном случае тривиальны, т.е. из тривиального закона сохранения с интегрирующим множителем $\Lambda=0$ следует, что

$$
\Psi^{\tau}\left(v, v_{y}\right)=v_{y}, \quad \Psi^{y}\left(v, v_{y}, v_{y y}, v_{3 y}\right)=-v_{3 y}+\frac{3}{2} v_{y}^{-1} v_{y y}^{2} \equiv-v_{\tau} .
$$

СлУчАй 1.2. Пусть $g=u$ с выражениями (3.2) для тока и потока. Уравнение (3.1) при этом приводит к

$$
v_{\tau}=v_{3 y}+\frac{3}{2} v^{-2} v_{y}^{3}-3 v^{-1} v_{y} v_{y y}
$$

Отметим, что это уравнение представляет собой специальный случай полулинейных эволюционных уравнений, которые допускают рекурсионный интегро-дифференциальный оператор, изученный в работе [16].

СлУчАй 1.3. Пусть $g=\ln (\alpha u) / \alpha$ с теми же током и потоком (3.2). Уравнение (3.1) при этом приводит к уравнению КдФ с модифицированным потенциалом:

$$
v_{\tau}=v_{3 y}+\lambda \alpha v_{y}^{3}, \quad \alpha^{2}=-2 \lambda .
$$

СлУчай 2. Рассмотрим $\Lambda_{2}=-2 u^{-3}$. Соответствующий не зависящий от $x$ ток нулевого порядка $\Phi^{t}$ и поток $\Phi^{x}$ для уравнения (3.1) имеют вид

$$
\Phi^{t}(u)=u^{-2}, \quad \Phi^{x}\left(u, u_{x}, u_{x x}\right)=2 u_{x x} .
$$

СлУчАЙ 2.1. Пусть $g=x$ с данными током и потоком (3.9). Преобразование $R$ принимает вид $x$-обобщенного преобразования годографа, а уравнение (3.1) при этом переходит в уравнение Кавальканте-Тененблата:

$$
v_{\tau}=v_{y}^{-3 / 2} v_{3 y}-\frac{3}{2} v_{y}^{-5 / 2} v_{y y}^{2} .
$$

СлУчАЙ 2.2. Пусть $g=u^{-1}$ с током и потоком (3.9). Преобразование $R$ при этом оказывается обратимым автопреобразованием Беклунда, так как при нем уравнение Гарри Дима переходит само в себя, т.е. в уравнение

$$
v_{\tau}=v^{3} v_{3 y}
$$

Это обратимое преобразование Беклунда было приведено в работе [17].

Случай 3. Рассмотрим $\Lambda_{3}=x u^{-3}$. Соответствующие ток нулевого порядка $\Phi^{t}$ и поток $\Phi^{x}$ для (3.1) имеют вид

$$
\Phi^{t}(x, u)=-\frac{1}{2} x u^{-2}, \quad \Phi^{x}\left(x, u, u_{x}, u_{x x}\right)=-x u_{x x}+u_{x} .
$$

Пусть $g=x$ с такими током и потоком. Получившееся обратимое преобразование Беклунда $R$ относится к классу $x$-обобщенных преобразований годографа. Оно переводит уравнение Гарри Дима (3.1) в уравнение

$$
v_{\tau}=v^{3 / 2} v_{y}^{-3 / 2} v_{3 y}+\frac{3}{2} v^{-1 / 2} v_{y}^{3 / 2}+\frac{3}{2} v^{3 / 2} v_{y}^{-5 / 2} v_{y y}^{2} .
$$


СлУчАЙ 4. Рассмотрим $\Lambda_{4}=-2 x^{2} u^{-3}$. Соответствующие ток нулевого порядка $\Phi^{t}$ и поток $\Phi^{x}$ для (3.1) имеют вид

$$
\Phi^{t}(x, u)=x^{2} u^{-2}, \quad \Phi^{x}\left(x, u, u_{x}, u_{x x}\right)=-2 x^{2} u_{x x}+4 x u_{x}-4 u .
$$

Пусть $g=x$ с указанными током и потоком. Получившееся обратимое преобразование Беклунда $R$ относится к классу $x$-обобщенных преобразований годографа. Оно переводит уравнение Гарри Дима (3.1) в уравнение

$$
v_{\tau}=v^{3} v_{y}^{-3 / 2} v_{3 y}-\frac{3}{2} v^{3} v_{y}^{-5 / 2} v_{y y}^{2} .
$$

СлучАЙ 5. Рассмотрим интегрирующий множитель

$$
\Lambda_{5}=-2\left(u^{-1} u_{x x}-\frac{1}{2} u^{-2} u_{x}^{2}\right) .
$$

СлУчАЙ 5.1. Отвечающий интегрирующему множителю (3.16) не зависящий от $x$ ток первого порядка $\Phi^{t}$ и поток $\Phi^{x}$ для (3.1) имеют вид

$$
\begin{aligned}
\Phi^{t}\left(u, u_{x}\right) & =u^{-1} u_{x}^{2} \\
\Phi^{x}\left(u, u_{x}, u_{x x}, u_{3 x}\right) & =-2\left(u^{2} u_{x} u_{3 x}+\frac{1}{2} u u_{x}^{2} u_{x x}-\frac{1}{8} u_{x}^{4}-\frac{1}{2} u^{2} u_{x x}^{2}\right) .
\end{aligned}
$$

Пусть $g(u)=u$ с данными током и потоком. Обратное преобразование Беклунда $R$ переводит уравнение Гарри Дима (3.1) в уравнение

$$
v_{\tau}=v^{6} v_{y}^{-6} v_{3 y}-3 v^{6} v_{y}^{-7} v_{y y}^{2}+3 v^{5} v_{y}^{-5} v_{y y}-\frac{3}{4} v^{4} v_{y}^{-3}
$$

СлУчАй 5.2. Отвечающий интегрирующему множителю (3.16) не зависящий от $x$ ток второго порядка $\Phi^{t}$ и поток $\Phi^{x}$ для (3.1) имеют вид

$$
\begin{aligned}
\Phi^{t}\left(u, u_{x}\right) & =-(\ln u) u_{x x} \\
\Phi^{x}\left(u, u_{x}, u_{x x}, u_{3 x}\right) & =\ln u\left(3 u^{2} u_{x} u_{3 x}+u^{3} u_{4 x}\right)+u^{2} u_{x x}^{2}-u u_{x}^{2} u_{x x}+\frac{1}{4} u_{x}^{4}-u^{2} u_{x} u_{3 x}
\end{aligned}
$$

Пусть $g(u)=u_{x}$ с этими током и потоком. Обратное преобразование Беклунда $R$ при этом переводит уравнение Гарри Дима (3.1) в уравнение

$$
v_{\tau}=v^{3} v_{y}^{6} v_{y y}^{-3} v_{3 y}-3 v^{3} v_{y}^{5} v_{y y}^{-1}+\frac{1}{4} v^{4} v_{y} .
$$

3.2. Уравнение Шварциана-КдФ. Рассмотрим уравнение Шварциана-КдФ в следующем виде:

$$
u_{t}=u_{x x x}-\frac{3}{2} u_{x}^{-1} u_{x x}^{2} .
$$


Полный класс не зависящих от $x$ и автономных присоединенных симметрий до порядка два включительно, которые также действуют как интегрирующие множители для уравнения (3.21), имеет вид

$$
\begin{aligned}
& \Lambda_{1}=\alpha\left(u^{2} u_{x}^{-3} u_{x x}-2 u u_{x}^{-1}\right), \\
& \Lambda_{2}=\alpha\left(u u_{x}^{-3} u_{x x}-u_{x}^{-1}\right), \\
& \Lambda_{3}=\alpha u_{x}^{-3} u_{x x}
\end{aligned}
$$

с произвольной константой $\alpha$. Для того чтобы вычислить токи $\Phi^{t}$ уравнения (3.21), рассмотрим два случая, а именно

$$
\begin{aligned}
& \Phi^{t}=\Phi^{t}\left(u, u_{x}\right), \\
& \Phi^{t}=\Phi^{t}\left(u, u_{x}, u_{x x}\right) .
\end{aligned}
$$

Ниже приводится список явных форм для $\Phi^{t}, \Phi^{x}$ и $g$ в обратимом преобразовании Беклунда $R$ вместе с получающимися при этом отображении уравнениями.

СлУчАй 1. Рассмотрим интегрирующий множитель $\Lambda_{1}$ из (3.22a). Если справедливо предположение (3.23a) с $\alpha=-2 / 3$, то мы получим

$$
\begin{aligned}
\Phi^{t}\left(u, u_{x}\right) & =\frac{1}{3} u^{2} u_{x}^{-1}, \\
\Phi^{x}\left(u, u_{x}, u_{x x}, u_{3 x}\right) & =\frac{1}{3} u^{2} u_{x}^{-2} u_{3 x}-\frac{1}{6} u^{2} u_{x}^{-3} u_{x x}^{2}-\frac{4}{3} u u_{x}^{-1} u_{x x}+\frac{4}{3} u_{x} .
\end{aligned}
$$

Обратное преобразование Беклунда $R(2.11)$ с $g(u)=u^{3}$ переводит (3.21) в уравнение

$$
v_{\tau}=v^{2} v_{y}^{-3 / 2} v_{3 y}-\frac{3}{2} v^{2} v_{y}^{-5 / 2} v_{y y}^{2}+\frac{4}{9} v_{y}^{3 / 2}
$$

Если справедливо предположение (3.23б) с $\alpha=-2 / 9$, то получим

$$
\begin{aligned}
\Phi^{t}\left(u, u_{x}, u_{x x}\right)= & \frac{2}{27} u^{3} u_{x}^{-3} u_{x x}, \\
\Phi^{x}\left(u, u_{x}, u_{x x}, u_{3 x}\right)= & -\frac{2}{27} u^{3} u_{x}^{-3} u_{4 x}+\frac{2}{9} u^{3} u_{x}^{-4} u_{x x} u_{3 x}+ \\
& +\frac{2}{9} u^{2} u_{x}^{-2} u_{3 x}-\frac{2}{9} u^{2} u_{x}^{-3} u_{x x}^{2}-\frac{1}{9} u^{3} u_{x}^{-5} u_{x x}^{3}-\frac{4}{9} u u_{x}^{-1} u_{x x}+\frac{4}{9} u_{x} .
\end{aligned}
$$

Обратное преобразование Беклунда $R(2.11)$ с $g\left(u, u_{x}\right)=u_{x}^{-2}$ переводит (3.21) в уравнение

$$
v_{\tau}=v^{-3 / 2} v_{y}^{4} v_{y y}^{-3} v_{3 y}+\frac{3}{2} v^{-5 / 2} v_{y}^{5} v_{y y}^{-2}-3 v^{-3 / 2} v_{y}^{3} v_{y y}^{-1}+\frac{4}{9} v^{-1 / 2} v_{y}
$$

СлУчАй 2. Рассмотрим интегрирующий множитель $\Lambda_{2}$, задаваемый формулой (3.22б). Если справедливо предположение (3.23a) с $\alpha=-2$, то мы имеем

$$
\begin{aligned}
\Phi^{t}\left(u, u_{x}\right) & =u u_{x}^{-1} \\
\Phi^{x}\left(u, u_{x}, u_{x x}, u_{3 x}\right) & =u u_{x}^{-2} u_{3 x}-\frac{1}{2} u u_{x}^{-3} u_{x x}^{2}-2 u_{x}^{-1} u_{x x} .
\end{aligned}
$$


Обратное преобразование Беклунда $R(2.11)$ с $g(u)=u$ переводит (3.21) в уравнение

$$
v_{\tau}=v^{3 / 2} v_{y}^{-3 / 2} v_{3 y}-\frac{3}{2} v^{3 / 2} v_{y}^{-5 / 2} v_{y y}^{2}-\frac{3}{2} v^{-1 / 2} v_{y}^{3 / 2} .
$$

Если справедливо предположение (3.23б) с $\alpha=1$, то мы получим

$$
\begin{aligned}
\Phi^{t}\left(u, u_{x}, u_{x x}\right)= & -\frac{1}{2} u^{2} u_{x}^{-3} u_{x x} \\
\Phi^{x}\left(u, u_{x}, u_{x x}, u_{3 x}\right)= & \frac{1}{2} u^{2} u_{x}^{-3} u_{4 x}-\frac{3}{2} u^{2} u_{x}^{-4} u_{x x} u_{3 x}-u u_{x}^{-2} u_{3 x}+u u_{x}^{-3} u_{x x}^{2}+ \\
& +\frac{3}{4} u^{2} u_{x}^{-5} u_{x x}^{3}+u_{x}^{-1} u_{x x} .
\end{aligned}
$$

Обратное преобразование Беклунда $R(2.11)$ с $g\left(u, u_{x}\right)=u_{x}^{-2}$ переводит (3.21) в уравнение

$$
v_{\tau}=v^{-3 / 2} v_{y}^{9 / 2} v_{y y}^{-3} v_{3 y}+\frac{3}{2} v^{-5 / 2} v_{y}^{11 / 2} v_{y y}^{-2}-3 v^{-3 / 2} v_{y}^{7 / 2} v_{y y}^{-1} .
$$

СлУчАй 3. Рассмотрим интегрирующий множитель $\Lambda_{3}$, задаваемый формулой (3.22в). Если справедливо предположение (3.23a) с $\alpha=-2$, то

$$
\begin{aligned}
\Phi^{t}\left(u, u_{x}\right) & =u_{x}^{-1} \\
\Phi^{x}\left(u, u_{x}, u_{x x}, u_{3 x}\right) & =u_{x}^{-2} u_{3 x}-\frac{1}{2} u_{x}^{-3} u_{x x}^{2} .
\end{aligned}
$$

Обратное преобразование Беклунда $R(2.11)$ с $g(u)=u$ переводит $(3.21)$ в уравнение

$$
v_{\tau}=v_{y}^{-3 / 2} v_{3 y}-\frac{3}{2} v_{y}^{-5 / 2} v_{y y}^{2} \text {. }
$$

Если справедливо предположение (3.23б) с $\alpha=-2$, то

$$
\begin{aligned}
\Phi^{t}\left(u, u_{x}, u_{x x}\right) & =2 u u_{x}^{-3} u_{x x} \\
\Phi^{x}\left(u, u_{x}, u_{x x}, u_{3 x}\right) & =-2 u u_{x}^{-3} u_{4 x}+6 u u_{x}^{-4} u_{x x} u_{3 x}+2 u_{x}^{-2} u_{3 x}-3 u u_{x}^{-5} u_{x x}^{3}-2 u_{x}^{-3} u_{x x}^{2} .
\end{aligned}
$$

Обратное преобразование Беклунда $R(2.11)$ с $g\left(u, u_{x}\right)=u_{x}^{-2}$ переводит (3.21) в уравнение

$$
v_{\tau}=v^{-3 / 2} v_{y}^{6} v_{y y}^{-3} v_{3 y}+\frac{3}{2} v^{-5 / 2} v_{y}^{7} v_{y y}^{-2}-3 v^{-3 / 2} v_{y}^{5} v_{y y}^{-1} .
$$

ЗАмечАниЕ. Напомним, что уравнение (3.28) также следует и из уравнения Гарри Дима (3.1) при $x$-обобщенном преобразовании годографа (см. (3.21)).

\section{4. ЗАКЛЮЧЕНИЕ}

Мы показали, как можно систематически использовать предложения 1 и 2 при построении обратимых преобразований Беклунда, задаваемых определением 1, для уравнений Гарри Дима и Шварциана-КдФ. Эти преобразования также включают в себя так называемое $x$-обобщенное преобразование годографа. Очевидно, что можно применить эту же процедуру к эволюционным уравнениям или к системам эволюционных уравнений, которые допускают интегрирующие множители, не зависящие 
от времени. Нужно тем не менее указать, что выбор функции $g$ в $R(2.11)$ должен быть таким, чтобы для преобразования $S$ существовала функция $h$, задаваемая в (2.13). Такой выбор обеспечивается предложением 2. Это не всегда возможно, если уравнения и системы уравнений имеют интегрирующие множители и законы сохранения только старших порядков. Расширенная классификация с применением этого подхода будет опубликована в отдельной работе.

Понятно, что если мы преобразуем симметрийно-интегрируемое эволюционное уравнение или систему эволюционных уравнений [11], применяя обратимое преобразование Беклунда, то получаемое эволюционное уравнение или система уравнений будет также обладать симметрийной интегрируемостью, т.е. допускать бесконечный набор высших законов сохранения и бесконечное число локальных симметрий Ли-Беклунда. Поскольку как уравнение Гарри Дима (3.1), так и уравнение Шварциана-КдФ (3.21) представляют собой симметрийно-интегрируемые уравнения, все уравнения, полученные выше, также оказываются симметрийно интегрируемыми эволюционными уравнениями. Тем не менее они не новы, поскольку полная классификация интегрируемых уравнений такого типа была приведена в работе [18]. Заметим, что в некоторых случаях необходимо произвести локальное преобразование для того, чтобы привести уравнения, рассмотренные в настоящей работе, к уравнению из работы [18]. В частности, полулинейное уравнение вида

$$
u_{t}=u_{x x x}+a\left(x, u, u_{x}\right) u_{x x}+b\left(x, u, u_{x}\right)
$$

приводится с помощью точечного преобразования к полулинейному уравнению $u_{t}=$ $u_{x x x}+f\left(x, u, u_{x}\right)$.

Отметим, что обобщения симметрийно-интегрируемых эволюционных уравнений третьего порядка типа Шварциана-КдФ (3.21) изучались недавно в связи с проблемой существования операторов рекурсии [19]; такие уравнения также включают в себя уравнения типа Кричевера-Новикова [20].

Благодарности. М. Эйлер и Н. Эйлер благодарны Swedish Research Council за оплату дорожных расходов, М. Эйлер благодарит Luleå University of Technology (грант № 2557-05) за финансовую поддержку, а С. Лундберг благодарит Royal Academy of Sciences (грант № FOA08V-032) за оплату дорожных расходов и Department of Mathematics at Luleå University of Technology за финансовую поддержку.

\section{Список литературы}

[1] C. Rogers, W. K. Schief, J. Nonlinear Math. Phys., 12, Suppl. 1 (2005), 548-564.

[2] A. Haar, Math. Ann., 100:1 (1928), 481-502.

[3] C. Rogers, W. F. Shadwick, Bäcklund Transformations and Their Applications, Math. Sci. Eng., 161, Academic Press, New York, 1982.

[4] A. S. Fokas, B. Fuchssteiner, Lett. Nuovo Cimento (2), 28:8 (1980), 299-303.

[5] A. M. Vinogradov, J. Math. Anal. Appl., 100:1 (1984), 1-40.

[6] A. M. Vinogradov, J. Math. Anal. Appl., 100:1 (1984), 41-129.

[7] В. В. Жаринов, ТМФ, 68:2 (1986), 163-171.

[8] V.V. Zharinov, Lecture Notes on Geometrical Aspects of Partial Differential Equations, Ser. Sov. East European Math., 9, World Sci., Singapore, 1992. 
[9] S. C. Anco, G. Bluman, European J. Appl. Math., 13:5 (2002), 545-566.

[10] S. C. Anco, G. Bluman, European J. Appl. Math., 13:5 (2002), 567-585.

[11] A.S. Fokas, Stud. Appl. Math., 77:3 (1987), 253-299.

[12] V.V. Sokolov, A. B. Shabat, "Classification of integrable evolution equations", Mathematical Physics Reviews, Soviet Sci. Rev. Sect. C Math. Phys. Rev., 4, ed. S. P. Novikov, Harwood Academic, Chur, 1984, 221-280.

[13] J. G. Kingston, C. Rogers, Phys. Lett. A, 92:6 (1982), 261-264.

[14] N. Euler, M. Euler, J. Nonlinear Math. Phys., 8:3 (2001), 342-362.

[15] M. Euler, N. Euler, N. Petersson, Stud. Appl. Math., 111:3 (2003), 315-337.

[16] N. Petersson, N. Euler, M. Euler, Stud. Appl. Math., 112:2 (2004), 201-225.

[17] J. G. Kingston, C. Rogers, D. Woodwall, J. Phys. A, 17:2 (1984), L35-L38.

[18] A. V. Mikhailov, A. B. Shabat, V. V. Sokolov, "The symmetrey approach to classification of integrable equations", What is Integrability?, Springer Ser. Nonlinear Dynam., ed. V. E. Zakharov, Springer, Berlin, 1991, 115-184.

[19] M. Euler, N. Euler, J. Nonlinear Math. Phys., 14:3 (2007), 313-315.

[20] M. Euler, J. Nonlinear Math. Phys., 15:2 (2008), 147-151. 